of galantamine in AD. The Galantamine USA10 Study Group. Neurology 2000;54:2269-76.

6. Wilkinson D, Murray J. Galantamine: a randomised, double-blind, dose comparison in patients with Alzheimer's disease. Int 7 Geriatr Psychiatry 2001;16:852-7.

7. Rockwood K, Mintzer J, Truyen L, Wessel T, Wilkinson D. Effects of a flexible galantamine dose in Alzheimer's disease: a randomised, controlled trial. 7 Neurol Neurosurg Psychiatry 2001, 71:589-95

8. Olin J, Schneider L. Galantamine for Alzheimer's disease [Cochrane review]. In: The Cochrane Library; Issue 4, 2001. Oxford: Update Software.

9. Wilcock G, Howe I, Coles H, Lilienfeld S, Truyen L, Zhu Y, et al. A long-term comparison of galantamine and donepezil in the treatment of Alzheimer's disease. Drugs Aging 2003;20:777-89.

Competing interests: The authors are employed within Johnson \& Johnson Pharmaceutical Services. Reminyl, a brand of galantamine, was developed by the research and development arm of Johnson \& Johnson and is marketed around the world by various operating companies of Johnson \& Johnson.

DOI:10.1053/cmaj.1031912

$\mathrm{A}^{\mathrm{lt}}$ though generally interesting, the meta-analysis by Krista Lanctôt and associates $^{1}$ of the efficacy and safety of cholinesterase inhibitors in the treatment of Alzheimer's disease simply demonstrates what is observed in day-to-day clinical practice: a slight improvement or stabilization of the disease (as indicated by neuropsychological evaluation) and various gastrointestinal side effects. The meta-analysis showed a slightly greater benefit with donepezil than with the other drugs - again, just what is observed in daily clinical practice.

Unfortunately, the extensive support that some of the authors received from the pharmaceutical industry, as outlined in the competing interests statement, ${ }^{1}$ might taint readers' perception of the authors' autonomy and independent interpretation of their findings. It is of course important to disclose potential competing interests, yet it is very difficult for readers to evaluate their influence on a study's conclusions. Furthermore, it might be useful if meta-analyses were to also include the competing interests of the authors of the papers included in the analysis. For example, the Japanese study ${ }^{2}$ that showed a substantial treatment effect with donepezil - ascribed in the meta-analysis ${ }^{1}$ to specific gene frequencies in the Japanese population - might have been influenced by the competing interests of its authors. As more and more clinical research is funded by companies with a financial interest in the outcomes, there may be greater pressure on researchers to assess and report their financial interests.

\section{Celio Levyman}

Neurologist

Saó Paulo, Brazil

\section{References}

1. Lanctôt KL, Herrmann N, Yau KK, Khan LR, Liu BA, LouLou MM, et al. Efficacy and safety of cholinesterase inhibitors in Alzheimer's disease: a meta-analysis. CMA7 2003;169(6):557-64.

2. Homma A, Takeda M, Imai Y, Udaka F, Hasegawa K, Kameyama M, et al. Clinical efficacy and safety of donepezil on cognitive and global function in patients with Alzheimer's disease. A 24-week, multicenter, double-blind, placebo-controlled study in Japan. E2020 Study Group. Dement Geriatr Cogn Disord 2000;11: 299-313.

Competing interests: None declared.

DOI:10.1053/cmaj.1031685

\section{[Three of the authors respond:]}

$\mathrm{T}$ he authors of these 2 letters raise several issues regarding our metaanalysis of the pivotal trials for 3 cholinesterase inhibitors used in the treatment of Alzheimer's disease.

Shane Kavanagh and Patricia Kabathova argue that the meta-analysis treats galantamine unfairly. In support of this claim, they point out that the majority of the galantamine trials included in the analysis did not use recommended doses. We explicitly recognized this and adjusted the meta-analysis of efficacy accordingly. They also suggest that metaanalyses focus on common treatment durations. However, there is no such thing as a "common treatment duration" in this context. In fact, the range of treatment duration is even greater than 12 weeks to 1 year, which Kavanagh and Kabathova refer to as "wide variation." We chose to be explicit about the treatment durations that were included and handled the differences by means of a subanalysis, assessing heterogeneity and providing confidence intervals.

In addition, Kavanagh and $\mathrm{Ka}-$ bathova suggest that more weight be given to the ADAS-cog assessment than to global clinical judgement. In keeping with the approach of health regulatory agencies such as the US Food and Drug
Administration, ${ }^{2}$ we provided the numbers needed to treat (NNT) and confidence intervals for both cognitive outcomes (the ADAS-cog scores) and global clinical impression. The emphasis on global outcomes was consistent with the stated goal of the analysis.

Kavanagh and Kabathova, Celio Levyman and other authors, ${ }^{3}$ all comment on the issue of comparability between the 3 drugs. However, in our meta-analysis we cautioned that individual cholinesterase inhibitors cannot be directly compared until welldesigned, properly blinded, head-tohead trials are conducted. Although Levyman shares his own clinical experience of a slightly greater benefit with donepezil, Kavanagh and Kabathova cite new evidence supporting the similarity in safety profiles between galantamine and other cholinesterase inhibitors. The study they selected as an example of a head-to-head trial ${ }^{4}$ was 1 of 3 published to date and was not available when we prepared our meta-analysis. All 3 studies $^{4-6}$ had relatively small numbers of subjects, and none was completely double-blinded. In each case, the results presented favoured the sponsor's drug. The similarities or differences among various cholinesterase inhibitors in terms of efficacy and safety remain to be established.

Levyman rightly points out that while the competing interests of the authors of the meta-analysis were fully disclosed, the primary randomized controlled trials used in the meta-analysis might themselves have been subject to bias. The bias we fear is the presentation of results unreasonably or unjustifiably favouring those of the sponsor or disfavouring those of a competitor. Although we cannot control for such bias, we did obtain and present results that were not distorted from what is found in clinical practice, that did not unduly favour the drug of any given sponsor and that did not unduly favour those drugs as a group or class. Bias also influences which trials are published, with negative trials less likely to be published. ${ }^{5}$ We addressed this potential problem by using funnel plots.

Meta-analysts and, more important, clinicians practising evidence-based 